\title{
Dipole formation at metal/PTCDA interfaces: role of the Charge Neutrality Level
}

\author{
H. Vázquez ${ }^{\dagger}$, R. Oszwaldowski ${ }^{\dagger}$, P. Pou ${ }^{\dagger}$, J. Ortega ${ }^{\dagger}$, R. Pérez ${ }^{\dagger}$, F. Flores ${ }^{\dagger}$ and A. Kahn \\ $\dagger$ Departamento de Física Teórica de la Materia Condensada- Universidad Autónoma de Madrid, E-28049 Madrid, Spain. \\ ฯ Department of Electrical Engineering- Princeton University, Princeton, NJ 08544, USA.
}

\begin{abstract}
The formation of a metal/PTCDA (3, 4, 9, 10- perylenetetracarboxylic dianhydride) interface barrier is analyzed using weak chemisorption theory. The electronic structure of the uncoupled PTCDA molecule and of the metal surface is calculated. Then, the induced density of interface states is obtained as a function of these two electronic structures and the interaction between both systems. This induced density of states is found to be large enough (even if the metal/PTCDA interaction is weak) for the definition of a Charge Neutrality Level for PTCDA, located $2.45 \mathrm{eV}$ above the highest occupied molecular orbital. We conclude that the metal/PTCDA interface molecular level alignment is due to the electrostatic dipole created by the charge transfer between the two solids.
\end{abstract}

Electronic materials made of molecular films are a fast developing field, with many potential applications in organicbased devices. Designing new organic-based materials requires a detailed understanding of the different processes occurring in these devices. In particular, metal/organic and semiconductor/organic interface barriers play a decisive role 1, 2]. However, the formation of barriers is not yet well understood.

In the Schottky-Mott model of metal/organic interfaces, it is assumed that no interface dipole is formed at the junction, and that the position of molecular levels with respect to the metal Fermi level is defined by vacuum level alignment. This situation was disproved by Narioka et al. [3] who, using ultra-violet photoemission spectroscopy (UPS), found large interface dipoles $(\sim 0.5-1.0 \mathrm{eV})$ at several metal/organic interfaces. Independent data by Hill et al. 4] confirmed this conclusion. Various mechanisms are believed to operate simultaneously at these interfaces, and several models have been advanced [1, 2]. Metal-molecule chemical reaction has been seen to create interface gap states that pin the Fermi level [5], a situation that is analogous to that described by the Unified Defect Model proposed for inorganic semiconductor/metal interfaces [6]. Compression of the metal surface electronic tail by adsorbed molecules, leading to vacuum level interface shift (the "pillow" effect), has also been proposed as a general metal/organic interface mechanism [7, 8, 9].

In this letter, we explore the first application to a metal/organic interface of the Induced Density of Interface (or virtual) States (IDIS) Model 10]. We study a metal/PTCDA (3, 4, 9, 10- perylenetetracarboxylic dianhydride) interface and analyze how the chemical interaction between the organic molecule and the metal creates an IDIS in the organic energy gap. Our calculations show that, although the chemical interaction is weak, the IDIS is large enough that a Charge Neutrality Level (CNL) of the organic molecule can be defined. Our results show that the interface Fermi level $E_{F}$ is pinned at the CNL, a situation similar to that described for the formation of Schottky barriers at conventional semiconductor/metal junctions.

In this theoretical analysis, we study the metal/PTCDA interaction in three steps. First, the molecular orbitals of the organic molecule are calculated using a Density Functional Theory (DFT) local-orbital method [1, 12]. Care is taken to identify the appropriate wavefunctions associated with the Affinity and Ionization levels defining the molecular transport gap. Then, we calculate the metal/molecule interactions and obtain the electronic induced density of states in the molecular energy gap. In this approach, we neglect the molecule-molecule interaction, which introduces only a small broadening of the molecular levels $(\sim 0.2 \mathrm{eV})$, and does not create any electron density of states in the molecular energy gap. Finally, we define the CNL associated with these induced density of states and show that the organic/metal interaction creates density of states large enough to pin the Fermi level close to the CNL. Pinning can be characterized by the parameter

$$
S=\frac{d E_{F}}{d \phi_{M}}
$$

(where $\phi_{M}$ is the metal workfunction), equivalent to the interface slope parameter in the theory of metal/semiconductor junctions [13] and which we find here to be $\sim 0.13$.

In our DFT study of the PTCDA molecule [12], we use an optimized minimal local orbital basis, and introduce many-body effects by means of a local orbital exchange and correlation potential [11]. In our actual calculations, we combined a local-orbital DFT code [14] with this exchange and correlation potential. This approach is equivalent to conventional DFT but is especially advantageous in cases where correlation is important [15], and for calculating the 


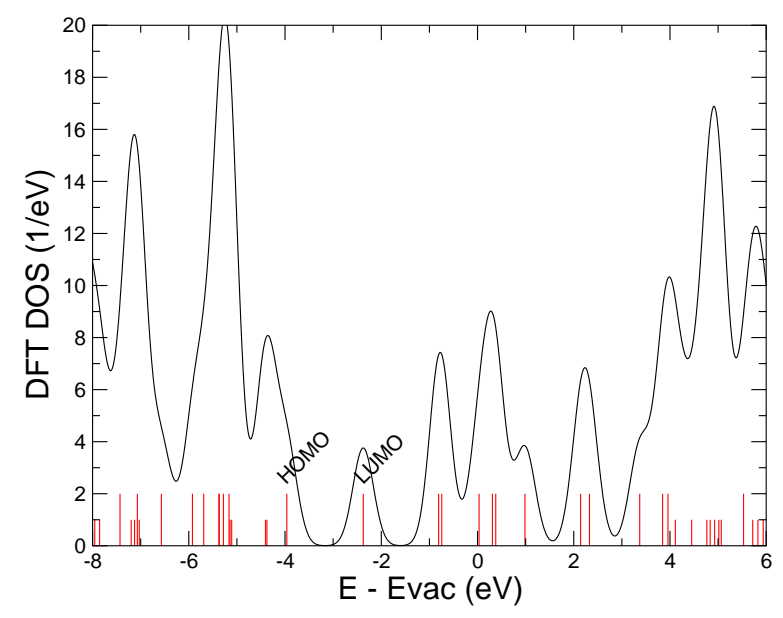

FIG. 1: DFT spectrum (long bars: $\pi$ states; short bars: $\sigma$ states) and DOS (introducing a 0.5 eV FWHM Gaussian broadening) for the PTCDA molecule.

Affinity $(A)$ and Ionization $(I)$ levels of the molecule, as will be seen below in the discussion of Koopman's theorem 12.

Figure 1 shows the spectrum obtained from the DFT calculation; we also calculate a LDOS by introducing for each level a Gaussian broadening with a $0.5 \mathrm{eV}$ FWHM [16]. This DFT calculation produces a molecular energy gap $\sim 1.6$ $\mathrm{eV}$, very small compared to the theoretical single-particle gap $A-I(\sim 5 \mathrm{eV})$ of the isolated molecule [17], and even smaller than its optical gap, $\sim 2.6 \mathrm{eV}[2]$. This is due to the fact that the DFT eigenvalues are not directly related to the molecular levels. Note also that, in analyzing the metal/organic interface, one needs to consider the single-particle energy gap of the isolated molecule, $A-I$. At the interface, this energy gap, $A-I$, is reduced by solid-state effects 2, 16, 18], as will be discussed later.

We first calculate $A$ and $I$ using the definitions $A=E[N+1]-E[N]$, and $I=E[N]-E[N-1]$, where $E\left[N_{i}\right]$ is the ground-state energy of the molecule with $N_{i}$ electrons. Each case can be calculated using the DFT method described above; we find [12] $I=-5.9 \mathrm{eV}$ and $A=-1.1 \mathrm{eV}$, yielding an energy gap of $4.8 \mathrm{eV}$, in reasonable agreement with the value of $\sim 5 \mathrm{eV}$ [17]. Interestingly, similar results can be obtained using a sort of Koopman's theorem [12], which amounts to introducing an electron (hole) in the LUMO (HOMO) of the neutral molecule, and calculating the total energy of the system neglecting electron relaxation effects. In this approach, the Affinity level, for instance, is simply the LUMO level corrected by a quantity $\delta A[12]$, which can be interpreted as a self-interaction energy associated with the new electron charge at the LUMO wavefunction. Likewise, the ionization-level correction, $\delta I$ [12], is a negative self-interaction energy related to the removal of one electron from the HOMO.

This Koopman's theorem approach yields $I=-6.1 \mathrm{eV}$ and $A=-1.2 \mathrm{eV}$, in very good agreement with the values given above. This also shows that electron relaxation effects in PTCDA associated with introducing or extracting an electron from the molecule are small, and indicates that the molecular orbitals calculated within DFT for the HOMO and LUMO levels are very appropriate for describing the Ionization and Affinity wavefunctions of the molecule. Note that $\pi$ and $\sigma$ orbitals give different values of $\delta A$ and $\delta I$. In our calculations, we obtain the PTCDA energy levels for the molecule by modifying the DFT $\pi$ and $\sigma$ levels with the corresponding values of $\delta A$ and $\delta I$ given by Koopman's theorem.

Solid-state effects associated with long-range electronic polarization have been analyzed by Tsiper et al. 18]. Polarization of molecules of the solid screens the electric field created by the extra charge introduced in the system, either an electron or a hole, modifying the energy levels $I$ and $A$ and the corresponding single-particle energy gap (also called transport gap in the solid). This correction is important, $1-1.5 \mathrm{eV}$, yet the ionization and affinity wavefunctions of the molecule are not expected to present important modifications. Other effects, such as lattice relaxation and vibronic coupling, only introduce corrections of the order of $0.2 \mathrm{eV}$, in the transport gap [16].

Thus, the electronic wavefunctions of PTCDA can be described using the results of a DFT approach, introducing a Koopman's correction for the $\pi$ and $\sigma$ molecular energy levels. Polarization effects are then included by fitting the ionization level and transport gap to their experimental values [19] by a rigid shift in the occupied and empty electron states. We fit the energy gap, $A-I$, to $3.2 \mathrm{eV}$, which seems to include all the effects discussed above.

We analyze the metal/PTCDA contact using chemisorption theory in the limit of weak interaction. The inset of Figure 3 shows the geometry of interest: a planar PTCDA molecule located at a distance $d$ from the outermost 


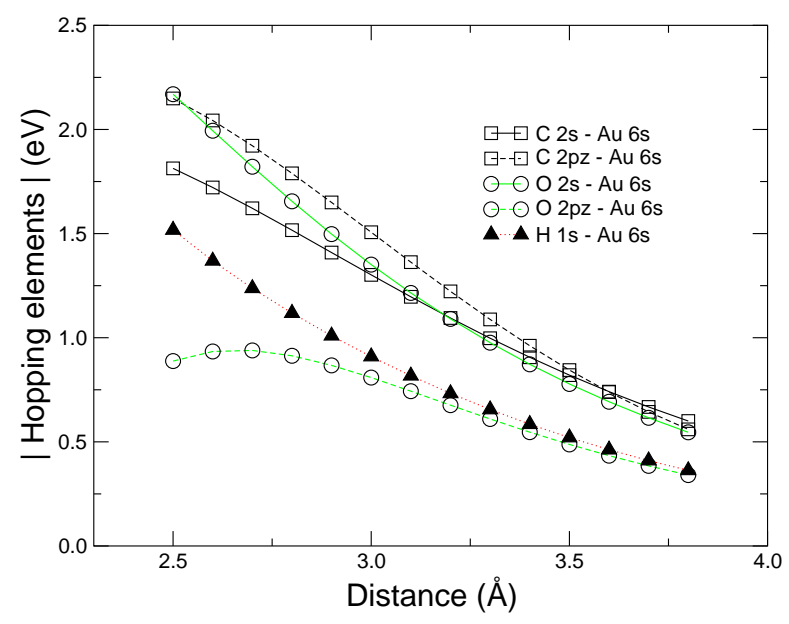

FIG. 2: $\mathrm{C}, \mathrm{O}$ and $\mathrm{H}-\mathrm{Au}$ interaction as a function of PTCDA-Au distance.

metal layer, i.e. the $\mathrm{Au}$ (111) surface. We assume the PTCDA wavefunctions, $\psi_{i}$, to be described by the DFT method discussed above, and the metal wavefunctions by a DFT-LDA local-orbital code [14], which also yields the Green-function, $G_{\alpha \beta}(E)$, where $\alpha$ and $\beta$ refer to the local-orbital basis used in the DFT code. In the limit of weak metal-PTCDA interacion [16], the main effect of the metal on the PTCDA electronic structure is to introduce the following self-energy for the molecular levels:

$$
\Sigma_{i}(E)=\sum_{\nu}\left|T_{i \nu}\right|^{2} G_{\nu}(E)
$$

where $T_{i \nu}$ is the hopping interaction between the molecular orbital $\psi_{i}$ and the metal eigenfunction, $\psi_{\nu}, G_{\nu}(E)$ being the corresponding Green-function. Equation 2 can be rewritten in a more appropriate way by using a local-orbital basis for the molecule and the metal. With the notation $\psi_{i}=\sum_{j} c_{i j} \phi_{j}$, equation 2 takes the form

$$
\Sigma_{i}(E)=\sum_{j j^{\prime} \alpha \beta} c_{i j} T_{j \alpha} G_{\alpha \beta}(E) T_{\beta j^{\prime}} c_{j^{\prime} i}
$$

where the self-energy of each molecular level is determined by: $(a)$ the hopping interaction $T_{j \alpha}$ between local orbitals; $(b)$ the coefficients $c_{i j}$ of the molecular orbitals $\psi_{i}$; and $(c)$ the Green-function of the metal, $G_{\alpha \beta}(E)$.

Figure 2 shows the hopping interaction between the Au $6 s$ orbital and the different orbitals of PTCDA, C $2 s$ and $2 p$, $\mathrm{O} 2 s$ and $2 p$, and $\mathrm{H} 1 s$ (in the following, we neglect the $5 d$ and $6 p$ orbitals of $\mathrm{Au}$, which is a reasonable approximation because we are interested in the induced density of states around the metal Fermi energy). These hopping elements are calculated using DFT for each pair of molecule-substrate atoms.

Equation 3 can be simplified to a more convenient form by neglecting contributions having $j \neq j^{\prime}$ : these terms represent interference effects between different local orbitals of PTCDA, and tend to cancel each other out. The contribution coming from the $\mathrm{Au} 6 s$ orbitals yields

$$
\Sigma_{i}(E)=\sum_{j, 6 s}\left|c_{i, j}\right|^{2}\left|T_{j, 6 s}\right|^{2} G_{6 s}(E)
$$

Each molecular level contributes to the LDOS with $-\frac{1}{\pi} \operatorname{Im}\left\{\frac{1}{\left(E-E_{i}\right)-\Sigma_{i}(E)}\right\}$. The main effect of $\Sigma_{i}$ is to broaden the molecular levels by $\operatorname{Im}\left(\Sigma_{i}(E)\right)$. Since we are studying the density of states around the molecular energy gap, we take $\Gamma_{i} / 2 \simeq \Sigma\left(E_{F}\right)\left(E_{F}\right.$ is the interface Fermi level $)$, and obtain

$$
\frac{1}{\pi} \frac{\Gamma_{i} / 2}{\left(E-E_{i}\right)^{2}+\left(\frac{\Gamma_{i}}{2}\right)^{2}}
$$




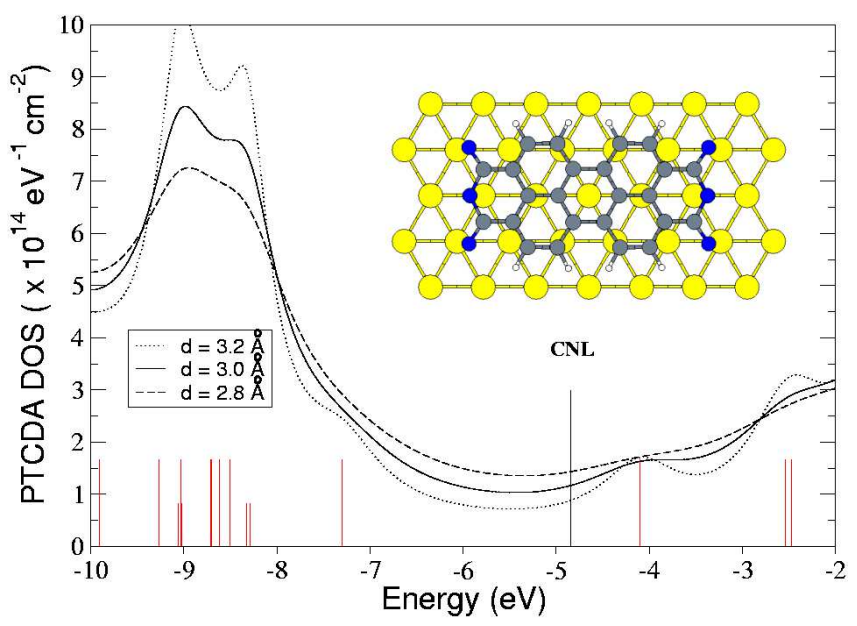

FIG. 3: PTCDA LDOS for the PTCDA/Au(111) interface (top view at inset) calculated for $d=2.8 \AA$ (dashed line), $d=3.0 \AA$ (continuous line), and $d=3.2 \AA$ (dotted line). Long (short) bars correspond to the $\pi(\sigma)$ states neglecting the metal-molecule interaction.

for the contribution of each molecular level to the LDOS.

As shown in Figure 2 $T_{j, 6 s}$ and $\Gamma_{i}$ depend on the PTCDA-Au distance, $d$. We do not know of any experimental value for this interfacial distance, nor are DFT calculations reliable in obtaining it, since the metal/PTCDA interaction has an important Van der Waals (or dispersion forces) contribution. Recently, Krause et al. [20] measured the PTCDA$\mathrm{Ag}(111)$ distance to be $\sim 2.85 \AA$, with an uncertainty of $\pm 0.2 \AA$. We expect a similar (or slightly larger) distance, of $\sim 3.0 \AA$ for the PTCDA-Au(111) system.

Figure 3 shows the PTCDA LDOS (both spins included) around the organic energy gap for $d=2.8,3.0$ and 3.2 $\AA$. For these distances, the $\pi$ and $\sigma$ levels are broadened by $\Gamma_{i}^{\pi} \simeq 2.1,1.5$ and $1.0 \mathrm{eV}$ and $\Gamma_{i}^{\sigma} \simeq 1.05,0.75$ and 0.5 $\mathrm{eV}$, respectively. The interactions $T_{j, 6 s}$ and the level broadening terms $\Gamma_{i}^{\pi}$ and $\Gamma_{i}^{\sigma}$ increase with decreasing distance: for $d=2.8 \AA$ the values of $\Gamma$ are typically twice the values for $d=3.2 \AA$. Figure 3 also shows the position of the CNL. The CNL is calculated by integrating the induced LDOS and imposing charge neutrality conditions: the total number of electrons up to the CNL equals that of the isolated molecule. The CNL is practically the same for the three distances $d=2.8,3.0$ and $3.2 \AA$, showing that it is a very robust quantity, practically independent of the details of the metal/PTCDA interaction.

We calculated the interface slope parameter, $S=\frac{d E_{F}}{d \phi_{M}}$. In our case [13],

$$
S=\frac{d E_{F}}{d \phi_{M}}=\frac{1}{1+4 \pi e^{2} D\left(E_{F}\right) \delta / A},
$$

where $D\left(E_{F}\right)$ is the PTCDA LDOS (Figure 3) at the Fermi level (practically, the CNL), $\delta$ is the distance between the charges induced in the metal and the organic molecule (between 2.8-3.2 $\AA$ in Figure 3) and $\mathrm{A}$ is the area associated with a PTCDA molecule (around $120 \AA^{2}$ ). We find a DOS of $1.5,1.2$ and $0.9 \times 10^{14} \mathrm{eV}^{-1} \mathrm{~cm}^{-2}$ for $d=2.8,3.0$ and 3.2 $\AA$, respectively. For these distances, $S \simeq 0.12,0.13$ and 0.16 . At variance with the CNL, $S$ strongly depends on the metal-PTCDA distance. But even for $d=3.5 \AA$, we obtain a DOS of $0.5 \times 10^{14} \mathrm{eV}^{-1} \mathrm{~cm}^{-2}$ and $\mathrm{S} \simeq 0.23$, indicating that the IDIS is still significant. This small value of $\mathrm{S}(0.1-0.2)$ shows that the LDOS induced in the molecule is high enough (even if the metal/PTCDA interaction is small) to strongly pin the interface Fermi energy close to the CNL.

Up to this point we have assumed the molecule to present no geometrical distortion when deposited flat on the metal. Recent DFT calculations for organic molecules adsorbed on metals [21] show non-negligible bond-length changes (e.g. the $\mathrm{C}-\mathrm{C}$ bond in an octane molecule adsorbed on $\mathrm{Cu}(110)$ is shortened by $\sim 0.04 \AA$ ). However, given the linear geometry of the alkanes, their geometrical distortions are probably larger than the ones in PTCDA on $\mathrm{Au}(111)$. Nevertheless, we explored the sensitivity of our results to geometrical distortions by changing some of the molecular bond lengths. We analyzed how the deformation of the molecular C-C and C-O bonds modify the position of the CNL and the LDOS. Our calculations indicate that the CNL shifts by $0.1 \mathrm{eV}$ for a change of $0.1 \AA$ of the carboxyl C-C bonds and by $\sim 0.07 \mathrm{eV}$ for a $0.1 \AA$ change in the $\mathrm{C}-\mathrm{O}$ bonds, while in all cases the LDOS around the CNL is practically unaltered. Thus, we conclude that the CNL undergoes only minor changes due to the molecular deformation, and that the LDOS is not significantly modified. The CNL shift due to molecular distortions can be 
estimated by realizing that the weaker single C-C bonds are probably going to suffer the largest deformation. In the case of the alkanes, these distortions are $\sim 0.05 \AA[21]$. This suggests that the accuracy in our calculated CNL, neglecting molecular deformations, is better than $\pm 0.1 \mathrm{eV}$.

From our calculations and the previous discussion, we find that the CNL is located $2.45 \pm 0.1 \mathrm{eV}$ above the center of the HOMO level of the molecule, in very good agreement with the experimental $E_{F}$ pinning position [4]. The value of $S \simeq 0.13$ is also in good agreement with $S \simeq 0$ inferred from experiments [4]. The important outcome of our analysis is that the calculated value of $S \simeq 0.13$ is indicative of a significant DOS at the metal/PTCDA interface, in spite of the weak chemical interaction between the two materials (see Figure 2). The implication is that the interface Fermi level is close to the CNL (in our calculations, $E_{F}$ lies $\sim 0.02 \mathrm{eV}$ below the CNL). Thus, the formation of the interface barrier is related to the transfer of charge across the interface. The charge transfer is associated with the IDIS, and creates an electrostatic interface dipole, which tends to align the metal Fermi level and the PTCDA CNL.

Recently, Bagus et al. 22] have suggested that for molecules physisorbed on metal surfaces a significant interface dipole, $\sim 0.3 \mathrm{eV}$, originates from exchange-like effects (this is the "pillow" effect mentioned above [7, 8, 9]). This effect can be easily incorporated in our discussion as a modification of the initial metal workfunction. As the final interface Fermi level $E_{F}$ is given by:

$$
\left(E_{F}-C N L\right)=S\left(\phi_{M}-C N L\right),
$$

where $E_{F}$, CNL and $\phi_{M}$ must have a common origin, a change of $0.3 \mathrm{eV}$ in $\phi_{M}$ is reflected in a Fermi level shift of $0.04 \mathrm{eV}(S=0.13)$. This suggests that for PTCDA, where $S$ is small, the "pillow" effect is almost negligible with regard to the final Fermi level position.

In conclusion, we have analyzed the metal/PTCDA interface barrier formation using weak chemisorption theory. The aim of this paper was to explore the importance of the IDIS and its contribution to the interface formation. Our results show that even for Au-PTCDA distances for which the interaction is weak $(d \sim 3.0-3.5 \AA)$, the IDIS is high enough to create an interface dipole that tends to align the Fermi level and the PTCDA CNL. A measure of this drive to align the two levels is given by the slope parameter, $S$, which our calculations show to be $\sim 0.13$.

Within this framework, we conclude that the main mechanism that leads to the formation of the Schottky barrier in metal/PTCDA interfaces is the charge transfer associated with the tunneling of metal electrons through the molecular energy gap. Our results show that the interface Fermi level should be close to the CNL of the molecule, which our calculations place $2.45 \pm 0.1 \mathrm{eV}$ above the HOMO level.

\section{Acknowledgments}

We gratefully acknowledge financial support by the Spanish CICYT under project MAT 2001-0665, the Comunidad de Madrid and the DIODE network (HPRN-CT-1999-00164). Support of this work by the National Science Foundation (DMR-0097133) and the New Jersey Center for Organic Optoelectronics (AK) is also acknowledged.

[1] See H. Ishii and K. Seki in "Conjugated Polymer and Molecular Interfaces", Ed. by W. Salaneck et al., Dekker (2001), p. 293-349.

[2] See C. Shen and A. Kahn in [1], p. 351-400.

[3] S. Narioka et al., Appl. Phys. Lett. 67, 1899 (1995).

[4] I. G. Hill et al., Appl. Phys. Lett. 73, 662 (1998).

[5] C. Shen, J. Schwartz and A. Kahn, J. Appl. Phys. 89, 449 (2001).

[6] W. E. Spicer et al., Appl. Surf. Sci. 41, 1 (1989).

[7] H. Ishii et al., Adv. Mater. 11, 605 (1999).

[8] A. Crispin et al., J. Am. Chem. Soc. 124, 8131 (2002).

[9] N. Koch et al., Appl. Phys. Lett. 82, 70 (2003).

[10] F. Flores and C. Tejedor, J. Phys. C 20, 145 (1987), W. Mönch, Surf. Sci. 299, 928 (1994).

[11] P. Pou et al., Phys. Rev. B 62, 4309 (2000).

[12] R Oszwaldowski et al., J. Phys. C 15, S2665 (2003).

[13] S. Z. Sze, "Physics of Semiconductor Devices", 2nd edition, Wiley, (1981).

[14] J. P. Lewis et al., Phys Rev B 64, 195103 (2001).

[15] P. Pou et al., Int. J. Quantum Chem. 91, 151 (2003).

[16] I. G. Hill et al., Chem. Phys. Lett. 317, 444 (2000). 
[17] M. Andrzejak, G. Mazur and P. Petelenz, J. Mol. Str. (Theochem) 527, 91 (2000).

[18] E. V. Tsiper et al., Chem. Phys. Lett. 360, 47 (2002).

[19] I. G. Hill et al., Chem. Phys. Lett. 327, 181 (2000).

[20] B. Krause et al., J. Chem. Phys. 119, 3429 (2003).

[21] H. Öström et al., J. Chem. Phys. 118, 3782 (2003).

[22] P. S. Bagus et al., Phys. Rev. Lett. 89, 096104 (2002). 